\title{
Política de Educação Permanente em Saúde: análise de sua implementação
}

\author{
Permanent Education in Health Policy: analysis of its implementation
}

Katerine Gonçalves Moraes ${ }^{1}$, Jane Lynn Garrison Dytz ${ }^{2}$

${ }^{1}$ Instituto de Atenção à Saúde São Francisco de Assis, Universidade Federal do Rio de Janeiro (HESFA/UFRJ); Policlínica Piquet Carneiro (PPC/UERJ) - Rio de Janeiro (RJ), Brasil.

${ }^{2}$ Programa de Pós-graduação em Enfermagem, Universidade de Brasília (UnB) - Brasília (DF), Brasil.

DOI: http://dx.doi.org/10.7322/abcshs.v40i3.806

\section{RESUMO}

Introdução: A política nacional de educação permanente em saúde define novas diretrizes para a gestão democrática na área da educação na saúde, tais como: a gestão participativa, o protagonismo dos trabalhadores e usuários e o fortalecimento do controle social. Objetivo: Analisar o processo de implementação da Política de Educação Permanente em Saúde no Distrito Federal, Brasil. Métodos: Estudo de caso utilizando a teoria da política pública foi realizado no Distrito Federal, envolvendo 24 participanteschave responsáveis pela gestão e implementação dessa política. Resultados: Os achados apontam para um modelo centralizado de gestão com pouca participação de outros segmentos da sociedade civil, obstáculos de financiamento devido a questões burocráticas e desvalorização dos espaços de execução da política. Conclusão: Pode-se concluir que as mudanças políticas e de gestão não têm sido realizadas com a amplitude necessária para a construção coletiva do conhecimento nos serviços de saúde.

Palavras-chave: política pública; recursos humanos; serviços de saúde; capacitação; educação continuada.

\begin{abstract}
Introduction: The national policy of permanent health education sets new guidelines for democratic management in the area of health education, such as: participatory management, the role of workers and users and the strengthening of social control. Objective: Analyze the implementation process of Permanent Education in Health Policy in Federal District, Brazil. Methods: A case study using the theory of public policy was held in the Federal District, involving 24 key participants responsible for the management and implementation of this policy. Results: The findings point to a centralized model of management with little involvement of other segments of the civil society, financing obstacles due to bureaucratic issues and devaluation of space policy implementation. Conclusion: It can be concluded that the policy changes and management have not been performed with the necessary breadth for the collective construction of knowledge in health services.
\end{abstract}

Keywords: public policy; human resources; health services; training; education, continuing. 


\section{INTRODUÇÃO}

A Política de Educação Permanente em Saúde (PEPS), instituída em 2004, é uma política pública estratégica que visa promover mudanças nos processos de formação e desenvolvimento dos trabalhadores do setor saúde, com base na problematização da realidade e na construção coletiva de soluções, tendo como foco as necessidades da população e o fortalecimento do Sistema Único de Saúde (SUS) ${ }^{1}$. Em 2007, a PEPS foi readequada, tendo em vista as mudanças no sistema de gestão na área da saúde, dando ênfase à descentralização e ao fortalecimento das instâncias gestoras e do controle social. A partir de então, os entes federados passaram a assumir a responsabilidade de criar estruturas de coordenação e execução em âmbitos regional e local ${ }^{2}$.

Analisando a evolução histórica desse tema, observa-se sua permanência como estado de coisas durante quase 18 anos. As conferências nacionais de saúde, apesar de constituírem-se lócus de definição de políticas públicas desde 1941, somente a partir de 1963 atuaram como espaço privilegiado dessa discussão. Observa-se, após análise dos relatórios das conferências, que, até o início da década de 1960, a questão do desenvolvimento de recursos humanos em saúde não fazia parte da agenda política, pois o foco estava em sanar a precária situação sanitária do país. Dos anos 1960 a 1980, as discussões se voltaram para aspectos relativos à qualificação profissional dos trabalhadores da saúde, principalmente os de baixa escolaridade ${ }^{3}$.

Somente a partir de 1986, durante a oitava conferência, que o desenvolvimento de recursos humanos para a saúde passou a se constituir pauta para a construção de políticas públicas, incluindo as questões relativas às condições laborais, iniciando as preocupações a respeito das relações precarizadas de trabalho e da necessidade de uma política de recursos humanos que também considerasse a gestão do trabalho no SUS. Em 2001, o Ministério da Saúde estabeleceu as diretrizes para a instituição de uma política nacional na área, ratificando a responsabilidade do SUS na ordenação da formação de recursos humanos para o setor ${ }^{4,5}$.

O foco do presente trabalho trata de um dos eixos da política o desenvolvimento dos trabalhadores já inseridos nos serviços -, tendo como pressuposto a relação entre educação e trabalho em saúde, cujo objetivo é induzir as mudanças necessárias no perfil dos profissionais e nas práticas de atenção e gestão dos serviços de saúde do SUS, fomentando o trabalho multiprofissional articulado às necessidades dos atores envolvidos ${ }^{6,7}$.

Tradicionalmente, as atividades educativas realizadas nos serviços de saúde eram organizadas tendo como base a proposta da educação continuada ${ }^{8}$. Miccas e Batista ${ }^{9}$, em recente metassíntese sobre o tema, destacam que a Educação Permanente em Saúde (EPS) foi apontada como sinônimo de Educação Continuada. Nessa proposta, a dimensão trabalhada diz respeito à atualização de saberes requerida pelos avanços técnicocientíficos, desenvolvida de forma pontual, fragmentada e com base em metodologias tradicionais ${ }^{9,10}$.

Motta et al. ${ }^{11}$ enfatizam que os avanços alcançados na gestão do sistema de saúde no Brasil tornaram necessária a revisão dos processos formativos em saúde, de forma que se orientassem pelos princípios e pelas diretrizes do SUS. Dessa forma, os autores criticam processos orientados apenas pela atualização técnico-científica, já que isolada ela não se mostra suficiente para alcançar essa dimensão de transformação imposta.

A diferença da proposta de educação permanente em saúde para as capacitações convencionais é que esta tem como ponto de partida o processo de trabalho e o protagonismo do trabalhador. As atividades educativas partem da problematização das práticas realizadas cotidianamente, com vistas à aprendizagem significativa e orientando-se para a melhoria dos serviços de saúde ${ }^{9,10,12}$.

Para consolidação dessa política, as diretrizes nacionais estabelecem que as propostas sejam construídas regionalmente, com base nas necessidades locais, e para isso devem levar em consideração a participação de atores estratégicos que conformam o quadrilátero da formação: ensino, gestão, atenção e controle social. A instância que possibilita a participação desses atores é a Comissão de Integração Ensino-Serviço (CIES) ${ }^{2,13}$.

Este estudo propõe trabalhar a etapa da implementação dessa política e optou-se como referencial teórico-metodológico a abordagem feita por dois estudiosos precursores na área da teoria da política pública: Pressman e Wildalvsky ${ }^{14}$. Eles partem do princípio de que a análise do processo de implementação de uma política é uma forma de fazer avaliação; sendo assim, para saber se uma política foi bem ou mal implementada, deve-se observar a diferença entre as consequências previstas com as de fato obtidas, mediante a análise de uma realidade concreta em sua totalidade.

Embora se saiba que a publicação de uma portaria não é garantia de que uma política pública seja implementada, é importante conhecer de que forma um ato administrativo governamental, no qual estão estabelecidas as diretrizes para um determinado assunto, chega até seu público-alvo e que grau de prioridade lhe é conferido pelas instâncias e pelos atores envolvidos.

Nesse sentido, a proposta do presente estudo é analisar o processo de implementação da política de educação permanente em saúde no Distrito Federal (DF), por meio do delineamento da estrutura institucional, dos espaços de articulação, do financiamento e dos atores responsáveis pela gestão da educação na saúde.

\section{MÉTODOS}

Trata-se de uma pesquisa qualitativa, do tipo estudo de caso, desenvolvida no DF de dezembro de 2011 a março de 2012. Por ser um território circunscrito, organizado em regiões administrativas subordinadas a um único gestor, constitui loco privilegiado para um estudo sobre implementação de política pública.

A gestão dos processos formativos e do desenvolvimento científico e tecnológico em saúde no DF é de responsabilidade da Fundação de Ensino e Pesquisa em Ciências da Saúde (FEPECS), instituição autônoma vinculada à Secretaria de Estado de Saúde (SES/DF). Subordinada a essa estrutura, até o ano de 2013, 
estava a Coordenação de Desenvolvimento de Pessoas (CODEP), à época responsável pela implementação da PEPS em toda a rede pública de saúde no DF, por meio da Gerência de Desenvolvimento de Projetos (GDP).

Na base do sistema estão 22 Núcleos de Educação Permanente em Saúde (NEPS), responsáveis pela organização e execução das ações pedagógicas nos serviços de saúde, estes ainda atuantes nas unidades. Destes, 15 estão localizados nas diretorias regionais de saúde e sete estão vinculados diretamente a serviços especializados ou fundações ligadas diretamente ao órgão gestor da SES/DF, sendo os responsáveis pelas atividades de educação na saúde de cerca de dezessete mil servidores distribuídos da seguinte forma: $60 \%$ são profissionais de nível fundamental/médio e 40\%, de nível superior, das diversas áreas em saúde ${ }^{15}$.

A pesquisa envolveu 24 informantes-chave, dos quais 21 eram chefes dos NEPS; apenas um não participou da pesquisa, pois o NEPS não estava em funcionamento no período de coleta de dados. Os outros três informantes ocupavam, à época da pesquisa, cargos de gestão na área de recursos humanos em saúde no governo do Distrito Federal: a coordenadora da CODEP, a gerente responsável pela GDP até dezembro de 2011 e a gerente que assumiu o cargo a partir de 2012.

A coleta de dados se deu por meio de entrevista semiestruturada, realizada no local de trabalho de cada sujeito, após obtenção de seu consentimento. As entrevistas foram organizadas e codificadas com o auxílio do software QSR Nvivo $8.0^{\circledR}$ e, em seguida, submetidas à técnica de análise de conteúdo temática proposta por Bardin ${ }^{16}$. A pesquisa foi aprovada pelo Comitê de Ética em Pesquisa da Fundação de Ensino e Pesquisa em Ciências da Saúde (FEPECS), da SES do Distrito Federal, sob o parecer $n^{\circ} 0231 / 2011$.

\section{RESULTADOS}

A análise de conteúdo dos relatos dos sujeitos possibilitou delinear a estrutura organizacional da gestão da educação na saúde no DF, bem como as arenas e os espaços de articulação, os atores responsáveis pela implementação da política distrital de educação permanente em saúde, suas estruturas de execução e as questões relativas ao financiamento.

A condução da gestão da educação na saúde na rede pública do DF envolve as seguintes arenas, que se constituem órgãos colegiados, e os seguintes atores:

1. Colegiado de Gestão Regional (CGR), composto pelos gestores na área da saúde.

2. Comissão Permanente de Integração EnsinoServiço (CIES), composta por 17 membros (gestores de saúde distritais, trabalhadores do SUS e/ou suas entidades representativas, instituições de ensino com cursos na área da saúde e movimentos sociais ligados à gestão das políticas públicas de saúde e do controle social no SUS). Não há gestor de educação ou seu representante, conforme previsto na política.
3. Coordenação de Desenvolvimento de Pessoas (CODEP) - sob a responsabilidade de uma enfermeira, sendo esse um cargo de indicação do gestor.

4. Conselho Distrital de Saúde - composto de 28 membros: 14 representantes de usuários, sete de gestores e sete de trabalhadores.

5. Comissão Intergestores Bipartite, composta pelos gestores distritais.

A Figura 1 apresenta o fluxo da gestão da educação na saúde na rede pública de saúde e as respectivas instâncias de pactuação.

Conforme se verifica, apesar de regularmente constituída e em funcionamento, a CIES não elabora o Plano de Ação Regional de Educação Permanente em Saúde (PAREPS) em conjunto com o Colegiado de Gestão Regional (CGR), tal como previsto na PEPS². Esse processo de trabalho é assumido pela própria CODEP que, após construir o PAREPS, o apresenta e pactua para a CIES, para o Conselho de Saúde do DF e, por fim, para o CGR para aprovação. Um dos sujeitos entrevistados abordou essa questão:

Quando ele (o PAREPS) passa pela CIES, ele tem que passar também pelo Conselho. Primeiro ele vem para a CIES que é mais específico, [...] então na CIES dá para a gente discutir essa questão mais específica, é tanto que a discussão é maior na CIES. No Conselho é 'isso é bom, isso não é bom'. Só que a gente tem que defender - isso aqui reforça o SUS, melhora para o usuário. $(\mathrm{C} 1)$

O Conselho Distrital de Saúde constitui-se em outra arena, sendo a CODEP membro efetivo dessa instância que conta com

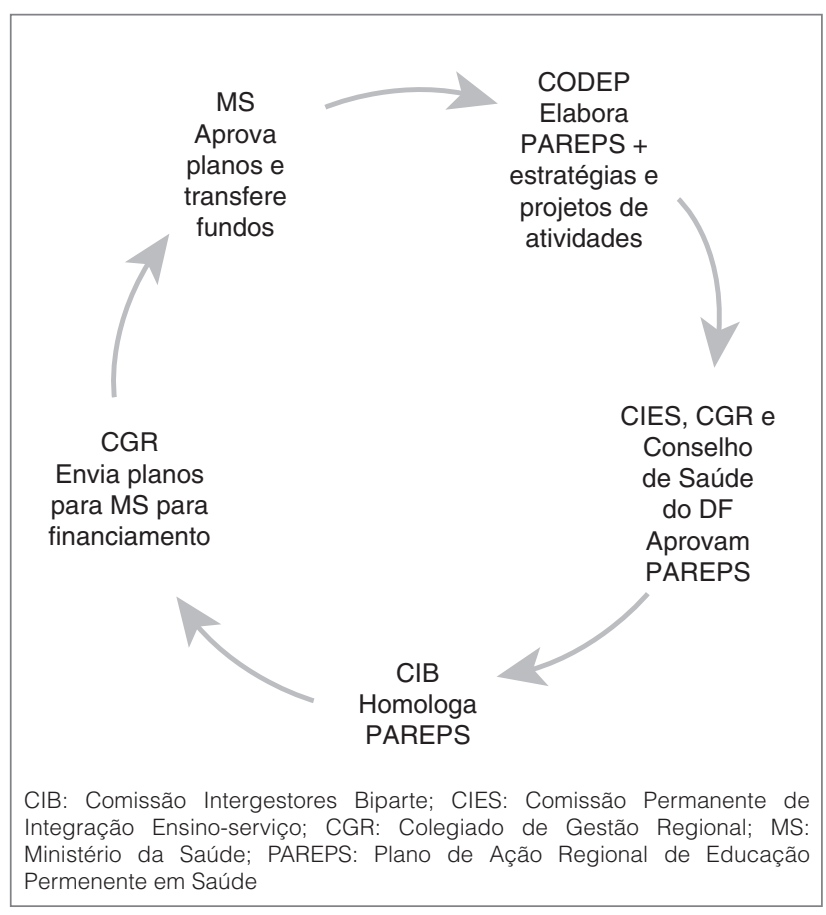

Figura 1: Fluxograma da gestão da educação na saúde na rede pública de saúde do Distrito Federal em 2012 
representantes da sociedade civil, podendo-se afirmar que ocorre certa articulação entre os gestores da política e os componentes do controle social. A fala abaixo ilustra esse ponto:

[...] ele passa também pela avaliação do Conselho, Conselho de Saúde, então eu acho que, assim, tendo em vista que o conselho é a representação de todas essas áreas, eu acho que, uma vez que ele é aprovado por lá, ele tem uma correlação com tudo. (C2)

A entrevistada enfatiza o papel dessa instância na aprovação dos PAREPS, cabendo a eles inclusive a reprovação dos projetos. $\operatorname{Cohn}^{17}$ afirma que aos Conselhos de Saúde cabe o exercício do controle social por meio da participação da sociedade, tal como previsto na legislação fundante do SUS. Segundo a autora, esse exercício ocorre por meio de deliberações no que diz respeito à definição de políticas de saúde e ao monitoramento de sua implementação. Essa arena de implementação constitui-se de fundamental importância para a efetiva participação social nas discussões da PEPS, permitindo um vínculo estreito entre as necessidades do SUS e as ações de educação na saúde propostas.

Os NEPS são as unidades executoras das atividades de educação na saúde na rede do SUS/DF. Segundo os participantes, os NEPS são caracterizados por estrutura física precária, insuficiência de recursos materiais e déficit em relação aos recursos humanos. Aliado a esses fatores, os relatos apontam para uma fragilidade na constituição das equipes de trabalho desses núcleos, sendo indicados pelos gestores dos serviços servidores que não têm formação adequada ou não se apropriam da complexidade inerente aos espaços de educação e saúde.

Ao realizar a indicação dos profissionais que irão constituir os NEPS, supõe-se que o gestor pretenda dar andamento às atividades daquela unidade, todavia, ocupar o espaço de implementação com atores alheios a esse processo é uma forma de dificultar a execução de uma política. Um sujeito abordou essa problemática:

[...] a antipenúltima, ela foi nomeada, foi um cargo político mesmo, e veio trabalhar no NEPS; quando eu cheguei, ela era que estava aqui, e ela não tinha noção nenhuma do que era educação, muito menos do que era saúde. (NS2)

Uma política pública envolve vários atores e níveis de decisão ${ }^{18}$; no caso da PEPS no DF, os chefes dos NEPS constituem-se em atores fundamentais, pois são eles os responsáveis pela implementação das atividades programadas no âmbito da política e cabe a eles a tomada de decisões no sentido da efetivação das metas propostas.

Ao serem indicados pelos gestores, no caso os diretores das instituições de saúde, sujeitos que não compreendem a importância da educação no trabalho em saúde podem se tornar um obstáculo a uma adequada implementação. Esse posicionamento do gestor não significa necessariamente que ele não concorde com a implementação da política, mas esta concorre com os mesmos recursos de que ele dispõe para efetivar outra ação, a saber, as atividades assistenciais nos serviços de saúde.

Para Pressman e Wildavsky ${ }^{14}$, determinados atores, no caso dessa política, os gestores, podem estar de acordo com os fins de uma determinada proposta, no entanto, podem se opor ou simplesmente deixar de facilitar os meios para realizá-la, sendo um dos motivos a preferência por outros programas.

Em relação ao seu financiamento, a educação na saúde no DF conta com duas fontes estatais: federal e distrital. O Ministério da Saúde libera anualmente recursos para a implementação das atividades previamente pactuadas nas arenas políticas referidas acima. Além desse recurso, a CODEP conta ainda com financiamento proveniente da FEPECS, fundação com orçamento próprio, vinculada diretamente à estrutura da SES. Essa questão foi discutida por um dos sujeitos que ocupava um cargo de gestão:

$\mathrm{Na}$ questão do financiamento, a gente tem mais uma parte complicada de orçamento que é: nós temos um orçamento que pertence à Fundação e a gente tem um recurso da educação permanente que é para capacitação e educação permanente [...] Esse é totalmente organizado por aqui, e nós temos também recurso que vem do Ministério da Saúde, que cai diretamente no fundo de saúde. Então, ele não vem para a Fundação, precisa ser uma negociação de transferência para cá ou uma negociação direta, que é o que a gente tem feito - preparar os processos aqui e encaminhar para a Secretaria pagar. (C1)

A questão relativa ao financiamento referida por um dos sujeitos está associada ao conflito de atribuições que ocorre na SES/ DF, pois esta conta em seu organograma com uma subsecretaria responsável pela gestão da educação na saúde, porém, essas ações têm sido realizadas historicamente por uma coordenação vinculada a uma fundação autônoma ligada à SES/DF. Ressalta-se que a subsecretaria não possui atribuições bem definidas nesse âmbito e o próprio plano de saúde do $\mathrm{DF}^{19}$ atribui a essa fundação a competência pela gestão da educação na saúde.

\section{DISCUSSÃO}

A análise da implementação da PEPS no DF teve como base a abordagem proposta por Pressman e Wildavsky ${ }^{14}$, na qual, durante o processo de implementação de uma política, os objetivos da política adaptam-se ao contexto, ajustando-se à rotina dos implementadores; disso resultam modificações entre os objetivos enunciados e as ações que de fato se efetivam. Nesse momento, torna-se crítico o papel dos atores envolvidos, no caso, os agentes que implementam a política e o público-alvo ao qual se destina ${ }^{20}$.

Contudo, o que se observa é que a implementação da PEPS não depende apenas da atitude de quem implementa, mas das condições disponíveis para a realização das atividades propostas para a obtenção do resultado almejado (Figura 2). 


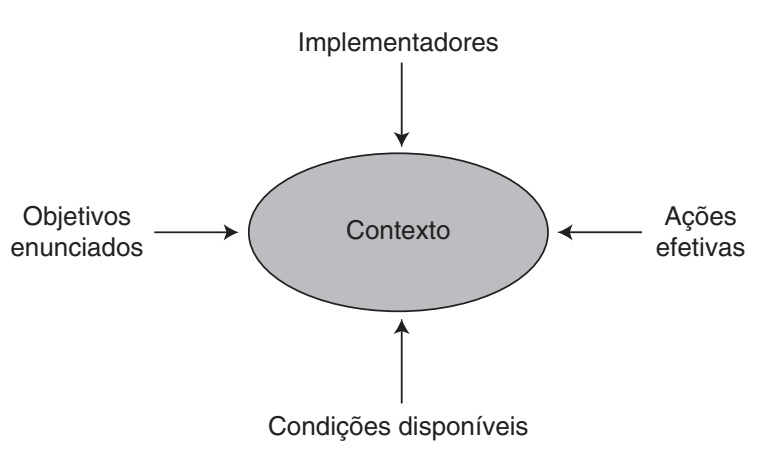

Figura 2: Fatores que interferem na implementação da Política de Educação Permanente em Saúde no Distrito Federal

Vale ressaltar que as etapas previstas em uma política para sua implementação não ocorrem de forma linear, pois dependem de circunstâncias que podem mudar, de metas que podem ser alteradas e das condições iniciais, tendentes a mutações. Dessa forma, não se pode prever essa sequência de acontecimentos, pois ela só se torna visível em meio à ação. Outro fato é que, quanto maior a cadeia de causas, mais numerosas serão as relações recíprocas que se estabelecem entre os elos e mais complexa será a implementação ${ }^{14}$.

Neste estudo, foram evidenciadas questões relacionadas aos aspectos formais da PEPS e que interferem diretamente na sua operacionalização, o que corrobora a forma de organização da educação na saúde no SUS/DF. Dentre as arenas envolvidas nessa política, destaca-se a CIES, que representa a imagem do quadrilátero da formação que integra ensino, serviço, gestão e controle social atuando de forma colegiada e em articulação interinstitucional. O objetivo é romper com a lógica verticalizada e hierarquizada e impor outra dinâmica aos processos organizativos ${ }^{13}$.

Entretanto, o que se observa é a pouca participação da CIES na construção das estratégias de intervenção na área da educação na saúde, sendo suas responsabilidades assumidas pela CODEP, que representa apenas o segmento gestor do quadrilátero, mantendo-se a lógica de centralização e verticalização na tomada de decisões.

No que diz respeito ao financiamento, observa-se que o recurso proveniente do Ministério da Saúde para a PEPS é transferido do fundo nacional para o fundo distrital de saúde, ficando sob a responsabilidade da SES/DF. Para que a unidade que coordena e executa tenha acesso a essa recurso, necessita elaborar projetos e encaminhá-los à SES para o financiamento.

Destaca-se que a descentralização orçamentária prevista na política foi resultado de uma ampla discussão em torno da Portaria $n^{\circ}$ 198/2004, que resultou na publicação da Portaria nº 1996/2007. Uma das mudanças feitas na PEPS a partir de 2007 foi desvincular o recurso financeiro da apresentação de projetos e possibilitar um mecanismo eficaz de descentralização financeira.

O que se observa no DF é uma centralização do recurso financeiro na SES, contudo, a CODEP e os NEPS são os responsáveis pela coordenação e execução das ações, tal como exposto anteriormente. Essa forma de financiamento, portanto, dificulta o acesso da CODEP aos recursos e, consequentemente, aos NEPS, pois há a necessidade de elaborar projetos.

Nesse sentido, vale questionar que lugar de prioridade na agenda do gestor distrital essa política ocupa, considerando a autonomia dos entes federados e a possibilidade de desenvolvimento das políticas a partir da sua esfera de atuação. O que se observa é que, apesar de dispor de recursos Federais e Distritais para a sua execução, existem trâmites burocráticos que dificultam a implementação das ações que são pensadas em âmbito local.

Segundo Pressman e Wildalvsky ${ }^{14}$, o número elevado de participantes ou protagonistas na implementação de uma política pública, como é o caso da PEPS, pode contribuir para que surjam desacordos e demoras, tendo em vista as diferentes perspectivas de cada ator e a multiplicidade de decisões.

Entretanto, o que se observou neste estudo foi uma centralização da construção das propostas da PEPS na figura do gestor e a baixa participação de outros segmentos, tais como o controle social, as instituições de ensino e os trabalhadores da saúde. Segundo os sujeitos, existe uma dificuldade para que a CIES funcione tal como explicitado na política, pois esses atores têm outras demandas provenientes das suas instituições de origem.

Tal fato minimiza a questão dos desacordos e demoras, mas, por outro lado, faz com que a implementação não ocorra como previsto nos instrumentos legais e não possibilite a participação de outros atores importantes na consolidação dessa proposta.

Nesse sentido, vale reconhecer que a manutenção burocrática de um espaço formal de encontro, no caso a CIES, não é suficiente para a produção das propostas que tenham como foco a educação permanente no cotidiano dos serviços; tampouco sem a participação ativa dos sujeitos que integram essa comissão haverá a concretização das ações que a política almeja.

Faz-se necessário ampliar as responsabilidades da CIES, não ficando apenas como instância que aprova ou não planos já construídos por atores que fazem parte da gestão, mas constituindo um processo de construção conjunta entre os sujeitos que a compõem.

Destaca-se a importância da participação social na construção da Política de Saúde no Brasil pelo papel que exerce na democratização da sociedade, por constituir-se um espaço institucional que possibilita aos setores organizados da sociedade civil exercerem o controle social sobre as ações do Estado na área da saúde, cabendo a eles deliberar sobre os recursos e sobre a política de saúde ${ }^{21}$.

Além da participação dos atores, existem questões administrativas que permeiam a implementação dessa política. A PEPS passa por diversas etapas, pois, após a aprovação dos PAREPS, cabe aos implementadores a construção de propostas de intervenção na área da educação na saúde. Pressman e Wildavsky ${ }^{14}$ alertam para a necessidade de políticas simples, pois, quanto menos etapas, menores as chances de falhas ou desvios.

Segundo os autores, políticas são sempre possibilidades. Nesse sentido, políticas potenciais dependem de suas qualidades intrínsecas e das circunstâncias externas para se tornar implementáveis. 
Nessa concepção, o PAREPS atua como instrumento (disposições e atitudes) para atingir os resultados esperados, desde que as circunstâncias sejam apropriadas. Assim, a implementação da PEPS não depende apenas de um plano bem elaborado, mas das circunstâncias externas que afetam este processo.

Nesse sentido, faz-se necessário estabelecer mecanismos de financiamento mais eficientes para essa política no DF de forma que viabilizem o acesso das estruturas de coordenação e execução aos recursos disponíveis e, como consequência, viabilize a operacionalização das atividades de educação permanente nos serviços de saúde do DF. Ressalta-se que essa discussão só será possível após o estabelecimento das atribuições quanto à gestão da educação na saúde da SES/DF.

Segundo Pressman e Wildavsky ${ }^{14}$, recursos insuficientes são fatores que podem colaborar para o fracasso na implementação de uma política, assim como a garantia de uma fonte de financiamento é considerada fator determinante para uma implementação exitosa.

Outra questão é a necessidade de objetivos claros e conceitos corretos, o que aumenta a probabilidade de uma implementação exitosa. A PEPS é clara em relação à definição de seus objetivos e conceitos, mas estes requerem mudanças complexas nos modos de organizar as ações de educação em saúde (bem como no próprio processo de trabalho), o que pode se tornar também fator dificultador para a implementação.

Destaca-se a existência de toda uma infraestrutura montada no sistema de saúde do DF para o trabalho de educação na saúde, bem como uma demanda crescente de qualificação profissional para melhoria da qualidade do atendimento na área da saúde; no entanto, constata-se um mau aproveitamento e uma desvalorização das arenas de implementação da política.

No que diz respeito à dimensão pedagógica, observa-se que a PEPS determina que as atividades educativas sejam desenvolvidas com base nos conceitos teórico-metodológicos da educação permanente em saúde. Dessa forma, tem como objetivo transformar as práticas nos serviços de saúde, por meio de um processo de aprendizagem significativa, a partir da análise coletiva dos processos de trabalho com a participação das equipes multidisciplinares.

Caracteriza-se, portanto, como uma política inovadora, com aspirações direcionadas para uma mudança de paradigma, já que implica não apenas na realização de atividades de educação em serviço, mas, principalmente, em instituir processos de mudanças na realidade dos serviços por meio da produção de conhecimento a partir do cotidiano e dos desafios enfrentados pelos trabalhadores diariamente, ou seja, o processo de trabalho em saúde é, em si, pedagógico. Essa característica torna a política complexa e pode dificultar sua implementação, pois visa a um envolvimento ativo dos diferentes atores na problematização da realidade vivida.

Após a análise da PEPS no DF, percebe-se que, apesar de não estarem sendo implementadas como preconizam as instruções normativas, as atividades propostas estão sendo realizadas rotineiramente nos serviços de saúde pelas estruturas de coordenação e execução.

Tal fato vai ao encontro do que afirmam Pressman e Wildavsky ${ }^{14}$ : para eles, quanto mais geral e mais adaptável for uma política a uma variedade de circunstâncias, maior a probabilidade de que se realize em certa forma, mas menos provável será que na prática surja como se havia pensado. Em contraponto, quanto mais se limita e mais se restringe a ideia da política, mais provável será que surja como se havia prognosticado, mas menos provável que se produza um impacto considerável.

Em um extremo, tem-se a ideia pré-formada de política como sendo um tipo ideal que só necessita executar-se e que os únicos problemas que suscita são os relativos ao seu controle. No outro extremo, a ideia da política é uma expressão de princípios e aspirações essenciais que dependem da discricionalidade administrativa, sendo ela inevitável e necessária para os processos de implementação.

Esta análise não tem como meta apenas realizar críticas aos problemas encontrados no processo de implementação da PEPS no DF, mas objetiva, por meio dos achados, buscar conhecer como se dá esse processo em uma realidade concreta e, a partir dessa aproximação, a possibilidade de encontrar soluções, por meio da elucidação dos problemas existentes.

O não alcance dos objetivos de uma política pode ser explicado por uma implementação defeituosa, ou seja, as atividades que deveriam acontecer não acontecem, ou pode-se considerar que as aspirações foram muito altas; dessa forma, em lugar de perguntar por que o processo de implementação foi defeituoso, a pergunta seria: por que se esperava tanto dele?

Ressalta-se que uma implementação perfeita de política pública é literalmente impossível, pois esta deve se dar sempre de forma evolutiva, aprendendo com a experiência e fazendo evoluir os problemas encontrados por meio da busca e implementação das soluções.

Para a implementação da PEPS, tal como explicitada nos documentos oficiais, será necessário uma mudança político-organizacional, de gestão e de paradigma, o que requer um longo processo de mudança cultural e social nas relações de trabalho e de vida dos atores envolvidos, já que ela apresenta metas ambiciosas que visam a mudanças nas práticas hegemônicas nos serviços de saúde. Estas só serão possíveis com a efetiva participação do controle social, dos trabalhadores da saúde e das instituições de ensino na construção e implementação das propostas.

Constata-se, portanto, que, para além do cumprimento dos trâmites burocráticos, a implementação dessa política pública depende da criação de estratégias que ampliem os espaços democráticos e de escuta dos trabalhadores e usuários e possibilitem o encontro e a mobilização dos diferentes atores na problematização do cotidiano, na identificação de problemas e na proposição de alternativas, investindo, assim, na sua capacidade de proposição e ação. Nesse sentido, sugere-se a realização de novos estudos que aprofundem o papel dos atores envolvidos nessa discussão. 


\section{REFERÊNCIAS}

1. Brasil. Ministério da Saúde. Portaria $n^{\circ}$ 198/GM/MS, de 13 de fevereiro de 2004. Institui a Política Nacional de Educação Permanente em Saúde como estratégia do Sistema Único de Saúde para a formação e o desenvolvimento de trabalhadores para o setor e dá outras providências. Brasília: Ministério da Saúde; 2004

2. Brasil. Secretaria de Gestão do Trabalho e da Educação na Saúde. Departamento de Gestão da Educação em Saúde. Política Nacional de Educação Permanente em Saúde. Brasília: Ministério da Saúde; 2009.

3. Moraes KG. Análise do Processo de Implementação da Política de Educação Permanente em Saúde no Distrito Federal. Dissertação (Mestrado) - Universidade de Brasília, Brasília, 2012.

4. Brasil. Ministério da Saúde. Conselho Nacional de Saúde. $11^{a}$ Conferência Nacional de Saúde. O Brasil falando como quer ser tratado: efetivando o SUS: acesso, qualidade e humanização na atenção à saúde com controle social: relatório final. Brasília: Ministério da Saúde; 2002.

5. Brasil. Ministério da Saúde [Internet]. Relatório final da $8^{\text {a }}$ Conferência Nacional de Saúde. 1986. Disponível em: http:// conselho.saude.gov.br/biblioteca/relatorios/relatorio_8.pdf. Acesso em: 03 abr. 2012

6. Pereira IB, Lima JCF. Trabalho, educação e saúde: referências e conceitos. In.: Pereira IB, Lima JCF. Dicionário da educação profissional em saúde. $2^{a}$ ed. Rio de Janeiro: ESPJV; 2009. p. 17-29

7. Silva LAA, Leite MT, Pinno C. Contribuições das comissões de integração ensino-serviço na educação permanente em saúde. Trab Educ Saúde. 2014;12(2):403-24 http://dx.doi.org/10.1590/S198177462014000200011

8. Costa PP. Dos projetos à política pública: reconstruindo a história da educação permanente em saúde. Dissertação (Mestrado) Fundação Oswaldo Cruz, Escola Nacional de Saúde Pública Sérgio Arouca, Rio de Janeiro, 2006.

9. Miccas FL, Batista SHSS. Educação permanente em saúde: metassíntese. Rev Saúde Pública. 2014;48(1):170-85. http://dx.doi.org/10.1590/S00348910.2014048004498

10. Falkenberg MB, Mendes TPL, Moraes EP, Souza EM. Educação em saúde e educação na saúde: conceitos e implicações para a saúde coletiva. Ciênc Saúde Coletiva. 2014;19(3):847-52. http://dx.doi.org/10.1590/141381232014193.01572013
11. Motta JIJ, Buss P, Nunes TCM. Novos desafios educacionais para a formação de recursos humanos em saúde. Olho Mágico. 2001;8(3).

12. Davini MC, Nervi L, Roschke MA. La capacitación del personal de los servicios de salud en proyectos relacionados con los procesos de reforma sectorial. Serie Observatorio de Recursos Humanos de Salud n.3. Programa de Desarrollo de Recursos Humanos (HSR). División de Desarrollo de Sistemas y Servicios de Salud (HSP). Organización Panamericana de la Salud, Organización Mundial de la Salud, 2002.

13. Ceccim RB, Feuerwerker LCM. O Quadrilátero da formação para a área da saúde: ensino, gestão, atenção e controle social. Physis. 2004;14(1):41-65 http://dx.doi.org/10.1590/S010373312004000100004

14. Pressman JL, Wildalvsky A. Implementation: How great expectations in Washington are dashed in Okland. In: Balboa AG, editor. Implementación: cómo grandes expectativas concebidas en Washington se frustan em Okland. México: Fundo de cultura econômica; 1998.

15. Brasil. Governo do Distrito Federal. Secretaria de Estado de Saúde do Distrito Federal [Internet]. Dados estatísticos - SES/DF. Dados estatísticos de serviços médicohospitalares. Disponível em: http:// www.saude.df.gov.br/sobreasecretaria/dadosestatisticos.html/. Acesso em: 28 ago. 2012.

16. Bardin L. Análise de conteúdo. Lisboa: Edições 70; 1977.

17. Cohn A. Cidadania e formas de responsabilização do Poder público e do setor privado pelo acesso, equidade, qualidade e humanização na atenção à saúde. Cad $11^{a}$ Conferência Nacional Saúde. 2000; p.43-55.

18. Souza C. Políticas públicas: uma revisão de literatura. Sociologias. 2006;8(16):20-45

19. Governo do Distrito Federal. Secretaria de Estado de Saúde do Distrito Federal. Plano de Saúde do Distrito Federal 2008 a 2011. Brasília: 2008.

20. Leite S, Flexor G, Medeiros L, Delgado NG, Maluf R. Avaliando a gestão de políticas agrícolas no Brasil: uma ênfase no papel dos policymakers. Rio de Janeiro: CPDA UFRRJ - CNPq; 2006

21. Correia MVC. Controle Social na Saúde. In: Mota AE, Bravo MIS, Uchôa R, editors. Serviço Social e Saúde: formação e trabalho profissional. $2^{\mathrm{a}}$ ed. São Paulo: Cortez, 2007. 